\title{
Magnetic-field Based Odometry - An Optical Flow Inspired Approach
}

Isaac Skog, Gustaf Hendeby and Felix Trulsson

The self-archived postprint version of this conference paper is available at Linköping University Institutional Repository (DiVA):

http://urn.kb.se/resolve?urn=urn:nbn:se:liu:diva-182273

N.B.: When citing this work, cite the original publication.

Skog, I., Hendeby, G., Trulsson, F., (2021), Magnetic-field Based Odometry - An Optical Flow Inspired Approach, Proceedings of the 2021 International Conference on Indoor Positioning and Indoor Navigation. https://doi.org/10.1109/IPIN51156.2021.9662626

Original publication available at:

https://doi.org/10.1109/IPIN51156.2021.9662626

Copyright: IEEE

http://www.ieee.org/

(C)2021 IEEE. Personal use of this material is permitted. However, permission to reprint/republish this material for advertising or promotional purposes or for creating new collective works for resale or redistribution to servers or lists, or to reuse any copyrighted component of this work in other works must be obtained from the IEEE. 


\section{Magnetic-field Based Odometry - An Optical Flow Inspired Approach}

\author{
Isaac Skog \\ Dept. of Electrical Engineering \\ Linköping University \\ Linköping, Sweden \\ isaac.skog@liu.se
}

\author{
Gustaf Hendeby \\ Dept. of Electrical Engineering \\ Linköping University \\ Linköping, Sweden \\ gustaf.hendeby@liu.se
}

\author{
Felix Trulsson \\ Dept. Underwater Technology \\ FOI Swedish Defence Research Agency \\ Kista, Sweden \\ felix.trulsson@foi.se
}

\begin{abstract}
An optical flow inspired magnetic-field based odometry estimation process is presented. The estimation process is based upon taking "image" like measurements of the magneticfield using a magnetometer array. From the measurements a model of the local field is learned. Using the learned model the pose change that gives the smallest prediction error of the measurement at the next time instant is calculated. Two models for describing the magnetic-field are presented, and the performance of the odometry estimation process when using the two models is evaluated. The evaluation shows that at a high signal-to-noise ratio the pose change can be estimated with an error of only a few percentage of the true pose change. Further, the evaluation shows that the uncertainty of the estimate can be consistently estimated. Thus, the proposed odometry estimation process can be used to reduce the navigation error growth rate of, for example, inertial navigation systems by providing reliable odometry information when passing by magnetized objects.
\end{abstract}

\section{INTRODUCTION}

Vector-fields, such as the earth's magnetic-field and gravityfield, are highly informative sources for localization. These exemplified vector-fields are omnipresent and stable, and variations in the fields, if measured accurately, provide a fingerprint highly correlated to the measurement location [1]; an example of the magnetic-field variations inside a building is shown in Fig. 1. Hence, these fields constitute a viable and robust information source for localization in Global Navigation Satellite System (GNSS) denied environments, such as indoors or underwater [2], [3]. Indeed, recent research on magneticfield based simultaneous localization and mapping (SLAM) using low-cost magnetometers and inertial sensors has shown impressive results and opened up a potential path toward scalable and accurate pedestrian indoor localization [4], [5]. However, a fundamental limitation of these magnetic-field based SLAM solutions is the requirement to frequently revisit mapped areas for mitigation of the inertial navigation errors. The allowable length of the exploratory phases depends on the uniqueness of the features and the navigation error growth rate of the inertial navigation process. Hence, to increase the usability of current magnetic-field based SLAM solutions there is a need for techniques that: (i) enable faster and more unique magnetic-field feature learning, and (ii) reduce the inertial navigation error growth rate. The latter is important not only in

978-1-6654-0402-0/21/\$31.00 (C) 2021 IEEE

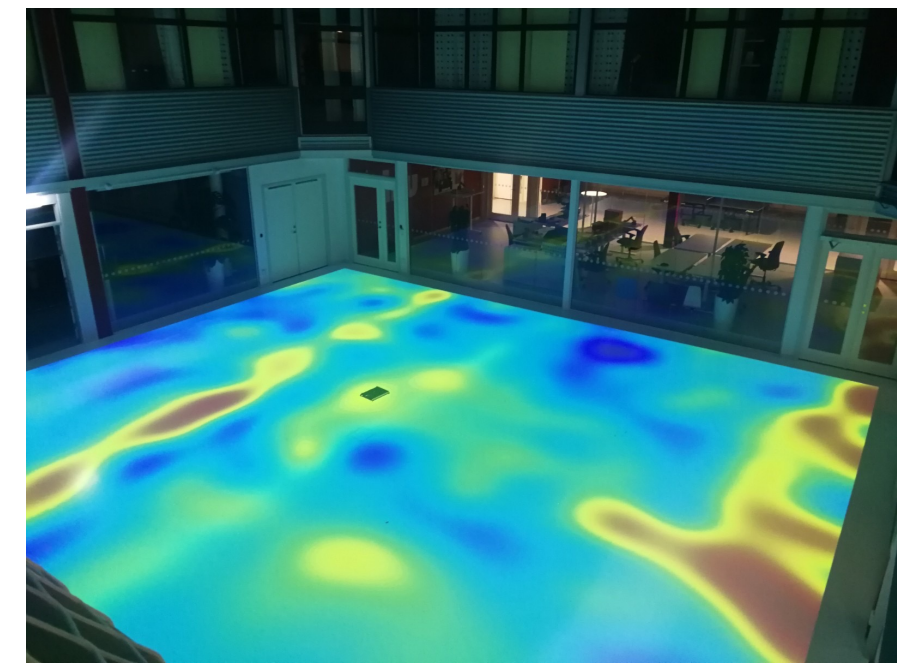

Fig. 1. Illustration of the magnetic-field magnitude variations inside a building. The field was measured with an magnetometer array, whose location was tracked by camera-based tracking systems. The field measurement was then interpolated and the field magnitude was projected on the floor.

magnetic-field based SLAM, but also for inertial sensor based navigation systems in general.

Today, thanks to the last decade's sensor technology development, high-performing and affordable magnetometer vectorsensor arrays can be constructed. Similarly to how a camera can take an image of the surrounding environment, these sensor arrays can take an image-like measurement of a vectorfield; see Fig. 2 for an example of a magnetic-field "image". And just as in computer-vision based localization systems, these magnetic-field measurements can be used for odometry and localization via feature tracking and feature matching. However, unlike visual imaging, magnetic-field imaging is not impaired by smoke, dust, fog, etc. Further, in contrast to the hard to model structure of visual images, the imaged magneticfield must comply with easy to model physical laws [6]. Therefore, this paper will present a model-based estimation method for magnetic-field "image" based odometry. 


\section{A. Related work}

The concept of magnet-field based odometry was first proposed in [7], where it was based upon the differential equation

$$
\frac{d m}{d t}=m \times \omega+\frac{d m}{d r} v
$$

The differential equation relates the rate of change of the magnetic field $m \in \mathbb{R}^{3}$ to the rotation rate $\omega \in \mathbb{R}^{3}$ (assumed to be measured by a gyroscope triad in [7]), the Jacobian of the magnetic field $d m / d r \in \mathbb{R}^{3 \times 3}$ with respect to the location $r \in \mathbb{R}^{3}$, and the velocity $v \in \mathbb{R}^{3}$. From the measurements of a magnetometer array the Jacobian $d m / d r$ can be estimated and the differential equation solved. That is, the velocity $v$ can be estimated.

In subsequent works [8] and [9] by the same authors, as well as in the recent papers [10]-[13], the differential equation (1) was used to develop magnetic-field based odometry aided inertial navigation system solutions. The result is a navigation system with a much slower error growth rate than a pure inertial navigation system; theoretically, the position error should grow linearly with time, instead of cubically. Indeed, the experimental results presented in [9] show that in an environment where there are sufficient variations in the magnetic field, such a magnetic odometry aided inertial navigation system can achieve a position error proportional to only a few percentage of the distance traveled.

Recently, a model-based approach to the magnetic-field odometry problem was proposed [14]. By introducing a model $\mathcal{M}$ of the local magnetic-field and fitting the model to two consecutive array measurements, i.e., two consecutive field images, the translational and rotational motion of the array between the measurements can be estimated. In relation to computer-vision odometry, the model-based approach can be viewed as performing sub-pixel interpolation using the model $\mathcal{M}$ and then doing optical-flow calculations for estimating the translational and rotational motion between the images [15].

Viewing the magnetic-field odometry problem as a model estimation problem has several benefits. First, estimation theory can be applied to analyze the properties of the magneticfield odometry problem and to derive various estimators. Second, the translational and rotational motion of the array can theoretically be estimated without any gyroscopes, which makes it possible to perform dead-reckoning using only an array of magnetometers. Finally, and possibly most importantly, the experimental results presented in [14] indicates that modelbased magnetic-field odometry may, at low signal-to-noise ratios, provide higher accuracy than odometry approaches based upon directly solving (1).

\section{B. Contributions}

Based upon the listed attractive properties of the modelbased odometry estimation process, we will in this paper extend the initial findings reported in [14] and present an optical flow inspired approach to magnetic-field odometry using magnetic-field "images". More precisely we will:

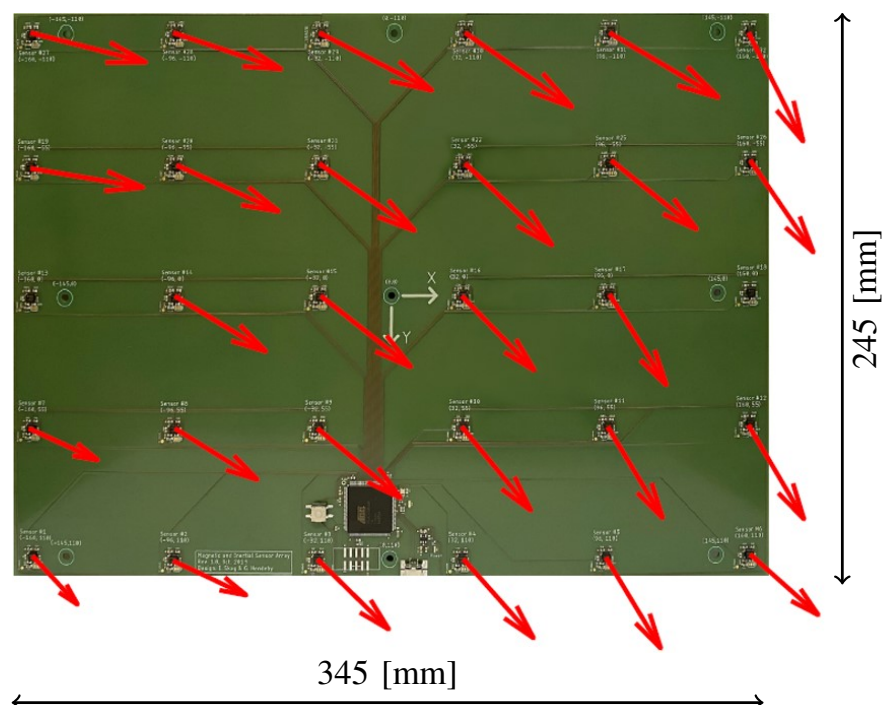

Fig. 2. Example of magnetic-field quiver plot, that is, a magnetic-field image, overlaid on the magnetic-field sensor array used to capture the field. Two arrows are missing due to broken sensors.

1) present two magnetic-field models suitable for modelbased magnetic-field odometry and describe how the parameters of these models can be learned; and

2) evaluate the odometry accuracy when using the two magnetic-field models to process magnetic-field measurements observed in a typical indoor environment.

All the data and code used to produce the presented results can be downloaded from www. openshoe. se.

\section{Proposed Odometry Estimation Process}

Next the proposed model-based odometry estimation process will be presented. To support the reader in assimilating the mathematical description of the estimation process a conceptional illustration of the process is presented in Fig. 3.

Consider a sensor array with $L \in \mathbb{N}$ three-axis magnetometers and let

$$
y_{k}=\left[\begin{array}{lll}
\left(y_{k}^{(1)}\right)^{\top} & \ldots & \left(y_{k}^{(L)}\right)^{\top}
\end{array}\right]^{\top}
$$

denote the measurements from these sensors at time instant $k \in \mathbb{N}$. Here $y_{k}^{(i)} \in \mathbb{R}^{3}$ denotes the measurement from the $i:$ th magnetometer in the array. Further, let the local magneticfield model $\mathcal{M}_{k}(r) \in \mathbb{R}^{3}$ be defined such that it describes the magnetic-field at location $r$ with respect to the array coordinate frame at time $k$. Moreover, let the pose change of the array between time instant $k$ and $k+1$ be denoted by

$$
x_{k+1}=\left[\begin{array}{ll}
\Delta p_{k+1}^{\top} & \Delta q_{k+1}^{\top}
\end{array}\right]^{\top} .
$$

Here $\Delta p_{k} \in \mathbb{R}^{3}$ and $\Delta q_{k} \in[0,2 \pi]^{3}$ denote the displacement and orientation change, respectively.

The array measurements at the next time instant, i.e., $y_{k+1}$, can then be modeled as

$$
y_{k+1}=h\left(x_{k+1} ; \mathcal{M}_{k}(r)\right)+e_{k+1}
$$


Field measurements at time instant: $k$
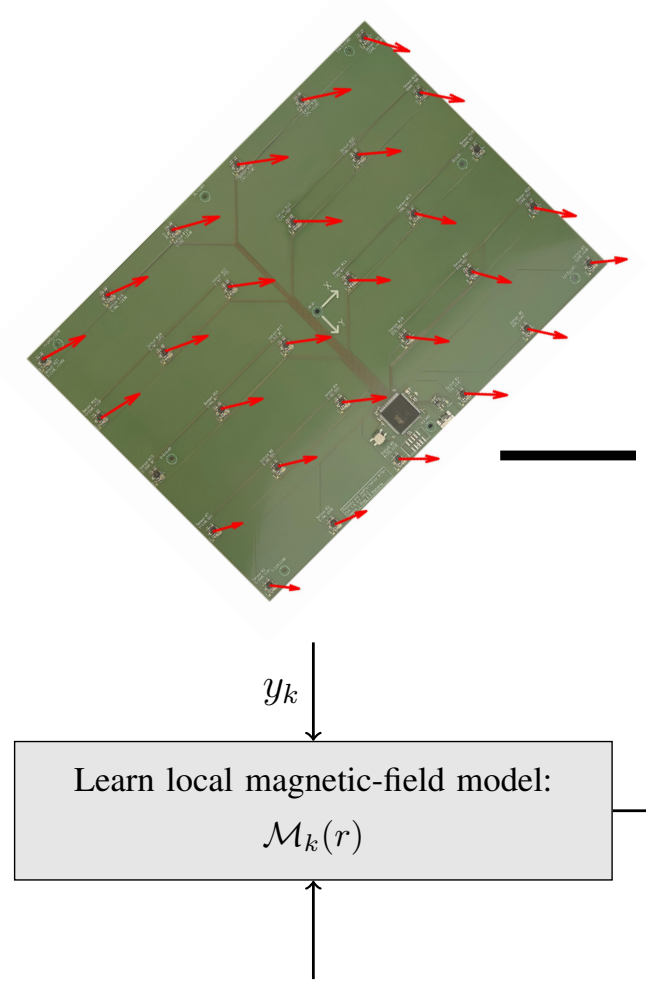

Input: Array geometry \& model type
Field measurements at time instant: $k+1$

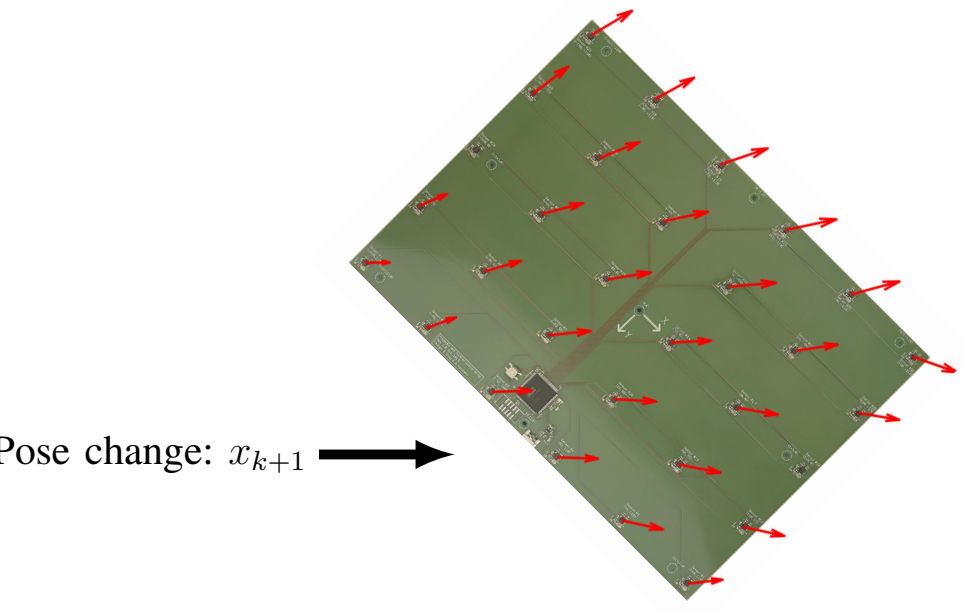

$y_{k+1} \downarrow$

Nonlinear least squares estimation: $\hat{x}_{k+1}=\arg \min _{x} V(x)$

Output: $\hat{x}_{k+1}, \Sigma_{\hat{x}_{k+1}}$

Fig. 3. Conceptional illustration of the proposed odometry estimation process. From the magnetic-field measurements $y_{k}$ a model $\mathcal{M}_{k}(r)$ of the local magnetic-field is learned. Using the learned model the pose change $\hat{x}_{k+1}$ that gives the smallest, in the weighted least squares sense, prediction error of the measurement $y_{k+1}$ is calculated. The covariance $\Sigma_{\hat{x}_{k+1}}$ of the pose change estimate is also calculated.

where

$$
h\left(x_{k+1} ; \mathcal{M}_{k}(r)\right)=\left[\begin{array}{c}
C\left(\Delta q_{k+1}\right) \mathcal{M}_{k}\left(r_{k+1}^{(1)}\right) \\
\vdots \\
C\left(\Delta q_{k+1}\right) \mathcal{M}_{k}\left(r_{k+1}^{(L)}\right)
\end{array}\right]
$$

and

$$
r_{k+1}^{(i)}=\Delta p_{k+1}+C\left(\Delta q_{k+1}\right) d^{(i)}
$$

Here $C\left(\Delta q_{k}\right) \in \mathbb{S O}(3)$ denotes the directional cosine matrix parameterized by $\Delta q_{k}$. Further, $d^{(i)} \in \mathbb{R}^{3}$ denotes the location of the $i$ :th magnetometer triad within the array. Moreover, $e_{k} \in$ $\mathbb{R}^{3 L}$ denotes the magnetometer array observation noise, which is assumed white with covariance $\Sigma_{e_{k}}=\sigma_{e}^{2} I_{3 L}$. Here $I_{3 L}$ $\left(0_{3 L}\right)$ denotes an identity (zero) matrix of dimension $3 L \times 3 L$.

In the definition of the measurement model (4a) it has been assumed that a perfect magnetic-field model $\mathcal{M}_{k}(r)$ is available. However, $\mathcal{M}_{k}(r)$ is typically unknown and must learned on the fly. Moreover, the learned model will generally not be perfect. If the model estimate $\hat{\mathcal{M}}_{k}(r)$ is unbiased and the model error small, then the error can be accounted for in the measurement model (4a) by substituting the observation noise covariance $\Sigma_{e_{k}}$ with the modified covariance

$$
\Sigma_{\varepsilon_{k}}=\left(I_{L} \otimes C\left(\Delta q_{k}\right)\right) \Sigma_{\hat{\mathcal{M}}_{k-1}}\left(I_{L} \otimes C\left(\Delta q_{k}\right)\right)^{\top}+\Sigma_{e_{k}}
$$

where

$$
\Sigma_{\hat{\mathcal{M}}_{k}}=\left[\begin{array}{ccc}
\Sigma_{\hat{\mathcal{M}}_{k}}^{(1,1)} & \ldots & \Sigma_{\hat{\mathcal{M}}_{k}}^{(1, L)} \\
\vdots & \ddots & \vdots \\
\Sigma_{\hat{\mathcal{M}}_{k}}^{(L, 1)} & \ldots & \Sigma_{\hat{\mathcal{M}}_{k}}^{(L, L)}
\end{array}\right]
$$

and

$$
\Sigma_{\hat{\mathcal{M}}_{k}}^{(i, j)}=\operatorname{Cov}\left\{\hat{\mathcal{M}}_{k}\left(r_{k+1}^{(i)}\right), \hat{\mathcal{M}}_{k}\left(r_{k+1}^{(j)}\right)\right\} .
$$

Note that the modified covariance matrix $\Sigma_{\varepsilon_{k}}$ depends on the pose change as it takes into account the uncertainties of the learned magnetic-field model $\hat{\mathcal{M}}_{k}(r)$ when extrapolating the measured field into new regions.

Thus, given a magnetic-field model estimate $\hat{\mathcal{M}}_{k}(r)$ and the measurement model (4a), the weighted least square estimate of the pose change $x_{k+1}$ can then be obtained as

$$
\hat{x}_{k+1}=\underset{x}{\arg \min } V(x)
$$

where

$$
V(x)=\left\|y_{k+1}-h\left(x ; \hat{\mathcal{M}}_{k}(r)\right)\right\|_{\Sigma_{\varepsilon_{k+1}}^{-1}(x)}^{2} .
$$

Further, the covariance of the estimate $\hat{x}_{k+1}$ is given by [16]

$$
\Sigma_{\hat{x}_{k+1}}=\hat{\lambda} H_{\hat{x}} \Sigma_{\varepsilon_{k+1}}^{-1}\left(\hat{x}_{k+1}\right) H_{\hat{x}}^{\top}
$$


where $\hat{\lambda}=V\left(\hat{x}_{k+1}\right) /(3 L)$ and

$$
H_{\hat{x}}=\left.\nabla_{x} h\left(x ; \hat{\mathcal{M}}_{k}(r)\right)\right|_{x=\hat{x}_{k+1}} .
$$

Next, two magnetic-field models $\mathcal{M}_{k}(r)$ and methods to learn the model parameters, will be presented.

\section{MAGNETIC-FIELD MODELS}

If the array moves within a static magnetic-field with no free current then, according to Maxwell's equations, the field should be both curl- and divergence-free [6]. Hence, the magnetic-field model $\mathcal{M}_{k}(r)$ should satisfy the conditions

$$
\begin{aligned}
\nabla_{r} \times \mathcal{M}_{k}(r) & =0 \\
\nabla_{r} \cdot \mathcal{M}_{k}(r) & =0
\end{aligned}
$$

Next, a polynomial and a Gaussian process model satisfying these conditions will be presented.

\section{A. Polynomial model}

A curl- and divergence-free polynomial magnetic-field model can be obtained by the linear model

$$
\mathcal{M}_{k}(r)=\Phi(r) \theta_{k}
$$

with the regression matrix $\Phi(r) \in \mathbb{R}^{3 \times \kappa}$ and parameter vector $\theta_{k} \in \mathbb{R}^{\kappa}$ selected as in [14]. For an $n$ :th order polynomial the model has $\kappa=\operatorname{dim}(\theta)=n^{2}+4 n+3$ unknown parameters. Since the model is linear the magnetic-field model can be estimated from the measurements $y_{k}$ as [16]

$$
\hat{\mathcal{M}}_{k}(r)=\Phi(r) \hat{\theta}_{k}
$$

where the model parameter estimate is given by

$$
\hat{\theta}_{k}=\left(\sum_{i=1}^{L} \Phi^{\top}\left(d^{(i)}\right) \Phi\left(d^{(i)}\right)\right)^{-1} \sum_{i=1}^{L} \Phi^{\top}\left(d^{(i)}\right) y_{k}^{(i)} .
$$

Further, the cross-covariance of the two estimates $\hat{\mathcal{M}}_{k}\left(r^{(i)}\right)$ and $\hat{\mathcal{M}}_{k}\left(r^{(j)}\right)$ is given by [16]

$$
\Sigma_{\hat{\mathcal{M}}}^{(i, j)}=\hat{\sigma}_{e}^{2} \Phi\left(r^{(i)}\right)\left(\sum_{i=1}^{L} \Phi^{\top}\left(d^{(i)}\right) \Phi\left(d^{(i)}\right)\right)^{-1} \Phi^{\top}\left(r^{(j)}\right)
$$

where

$$
\hat{\sigma}_{e}^{2}=\frac{1}{3 L} \sum_{i=1}^{L}\left\|y_{k}^{(i)}-\Phi\left(d^{(i)}\right) \hat{\theta}_{k}\right\|^{2}
$$

The polynomial order, i.e., $n$, can be selected using, e.g., the Akaike information criterion (AIC) [17]. Further, the computational complexity of the learning process is approximately $n^{4} L^{2}$.

\section{B. Gaussian process model}

A non-parametric method for modeling magnetic-fields using a curl- and divergence-free Gaussian process was presented in [6]. Adapted to the notation used within this paper, the method can be summarized as follows. The magnetic-field and the magnetization of the material in which the field is observed can jointly be modeled as

$$
f(r)=\left[\begin{array}{c}
\mathcal{M}(r) \\
\eta(r)
\end{array}\right] \sim \mathcal{G P}\left(0, K\left(r, r^{\prime}\right)\right)
$$

Here $\eta(r) \in \mathbb{R}^{3}$ denotes the magnetization of the material and $\mathcal{G} \mathcal{P}\left(0, K\left(r, r^{\prime}\right)\right)$ denotes a zero-mean Gaussian process with kernel function $K\left(r, r^{\prime}\right) \in \mathbb{R}^{6 \times 6}$. The kernel function describes the correlation of the Gaussian process between point $r$ and point $r^{\prime}$, i.e., $K\left(r, r^{\prime}\right)=\operatorname{Cov}\left\{f(r), f\left(r^{\prime}\right)\right\}$, and is in the considered case defined as

$$
K\left(r, r^{\prime}\right)=\left[\begin{array}{cc}
K_{B}\left(r, r^{\prime}\right) & K_{B}\left(r, r^{\prime}\right) \\
K_{B}\left(r, r^{\prime}\right) & K_{B}\left(r, r^{\prime}\right)+K_{H}\left(r, r^{\prime}\right)
\end{array}\right]
$$

where

$$
\begin{aligned}
K_{B}\left(r, r^{\prime}\right) & =\sigma_{\operatorname{lin}}^{2} I_{3}+\sigma_{f}^{2} e^{-\frac{\left\|r-r^{\prime}\right\|^{2}}{2 \ell^{2}}} \\
& \cdot\left(\frac{\left(r-r^{\prime}\right)\left(r-r^{\prime}\right)^{\top}}{\ell^{2}}+\left(2-\frac{\left\|r-r^{\prime}\right\|^{2}}{\ell^{2}}\right) I_{3}\right)
\end{aligned}
$$

and

$$
\begin{aligned}
K_{H}\left(r, r^{\prime}\right) & =\sigma_{\operatorname{lin}}^{2} I_{3}+\sigma_{f}^{2} e^{-\frac{\left\|r-r^{\prime}\right\|^{2}}{2 \ell^{2}}} \\
& \cdot\left(I_{3}-\frac{\left(r-r^{\prime}\right)\left(r-r^{\prime}\right)^{\top}}{\ell^{2}}\right) .
\end{aligned}
$$

Here, $\sigma_{f} \in \mathbb{R}^{+}$and $\ell \in \mathbb{R}^{+}$denote the magnitude variations and the length scale of the radial base function part of the kernel. Moreover, $\sigma_{\text {lin }} \in \mathbb{R}^{+}$denotes the magnitude variations of the linear part of the kernel. These three hyperparameters can either be set based upon prior knowledge about the underlying magnetic-field or learned from the field observations as described in [18].

Given the Gaussian process model in (12) the magnetic field $\mathcal{M}_{k}(r)$ at the locations $r_{k+1}^{(i)}, i=1, \ldots, L$ can be predicted from the measurements $y_{k}$ via the following four steps. First, define the two vectors

$$
f^{*}=\left[\begin{array}{c}
\mathcal{M}\left(r_{k+1}^{(1)}\right) \\
\vdots \\
\mathcal{M}\left(r_{k+1}^{(L)}\right) \\
\eta\left(r_{k+1}^{(1)}\right) \\
\vdots \\
\eta\left(r_{k+1}^{(L)}\right)
\end{array}\right] \quad \text { and } \quad f^{\circ}=\left[\begin{array}{c}
\mathcal{M}\left(d^{(1)}\right) \\
\vdots \\
\mathcal{M}\left(d^{(L)}\right) \\
\eta\left(d^{(1)}\right) \\
\vdots \\
\eta\left(d^{(L)}\right)
\end{array}\right]
$$

describing the magnetic-field and magnetization at the prediction and measurement location, respectively. Further, in- 
troduce the ideal pseudo magnetization measurement vectors $y_{k}^{\eta} \in \mathbb{R}^{3 L}$ and $y_{k+1}^{\eta} \in \mathbb{R}^{3 L}$ defined as

$$
y_{k}^{\eta}=\left[\begin{array}{c}
\eta\left(d^{(1)}\right) \\
\vdots \\
\eta\left(d^{(L)}\right)
\end{array}\right] \quad \text { and } \quad y_{k+1}^{\eta}=\left[\begin{array}{c}
\eta\left(r_{k}^{(1)}\right) \\
\vdots \\
\eta\left(r_{k}^{(L)}\right)
\end{array}\right]
$$

respectively. Moreover, let $y_{k}^{\eta}=0$ and $y_{k+1}^{\eta}=0$ since the magnetization at all physically feasible locations of the array must be zero. ${ }^{1}$

Second, assume that the measurement noise $e_{k}$ is Gaussian distributed and calculate the posterior mean and covariance of $f^{*}$ given the measurements $y_{k}$ and $y_{k}^{\eta}$. That is, calculate [18]

$$
\begin{aligned}
\mu_{f^{*} \mid y_{k}, y_{k}^{\eta}} & =\mathrm{E}\left\{f^{*} \mid y_{k}, y_{k}^{\eta}\right\} \\
& =\Sigma_{f^{*} f^{\circ}}\left(\Sigma_{f^{\circ} f^{\circ}}+\Sigma_{\dot{e}}\right)^{-1}\left[\begin{array}{c}
y_{k} \\
y_{k}^{\eta}
\end{array}\right]
\end{aligned}
$$

and

$$
\begin{aligned}
\Sigma_{f^{*} f^{*} \mid y_{k}, y_{k}^{\eta}} & =\operatorname{Cov}\left\{f^{*}, f^{*} \mid y_{k}, y_{k}^{\eta}\right\} \\
& =\Sigma_{f^{*} f^{*}}-\Sigma_{f^{*} f^{\circ}}\left(\Sigma_{f^{\circ} f^{\circ}}+\Sigma_{\dot{e}}\right)^{-1} \Sigma_{f^{*} f^{\circ}}^{\top},
\end{aligned}
$$

where the covariance matrices $\Sigma_{f^{*} f^{*}}=\operatorname{Cov}\left\{f^{*}, f^{*}\right\}, \Sigma_{f^{*} f^{\circ}}=$ $\operatorname{Cov}\left\{f^{*}, f^{\circ}\right\}, \Sigma_{f^{\circ} f^{\circ}}=\operatorname{Cov}\left\{f^{\circ}, f^{\circ}\right\}$, and $\Sigma_{\dot{e}}=\Sigma_{e} \oplus 0_{3 L}$ can be calculated using the kernel definition in (13a).

Third, update the posterior mean and covariance using the knowledge that $y_{k+1}^{\eta}$ also should be zero. That is,

$$
\begin{array}{r}
\mu_{f^{*} \mid y_{k}, y_{k}^{\eta}, y_{k+1}^{\eta}}=\mathrm{E}\left\{f^{*} \mid y_{k}, y_{k}^{\eta}, y_{k+1}^{\eta}\right\} \\
\left(I_{6 L}-G\right) \mu_{f^{*} \mid y_{k}, y_{k}^{\eta}}
\end{array}
$$

and

$$
\begin{aligned}
\Sigma_{f^{*} f^{*} \mid y_{k}, y_{k}^{\eta}, y_{k+1}^{\eta}} & =\operatorname{Cov}\left\{f^{*}, f^{*} \mid y_{k}, y_{k}^{\eta}, y_{k+1}^{\eta}\right\} \\
& =\left(I_{6 L}-G\right) \Sigma_{f^{*} f^{*} \mid y_{k}, y_{k}^{\eta}}
\end{aligned}
$$

where

$$
\begin{aligned}
G & =\Sigma_{f^{*} f^{*} \mid y_{k}, y_{k}^{\eta}} U^{\top}\left(U \Sigma_{f^{*} f^{*} \mid y_{k}, y_{k}^{\eta}} U^{\top}\right)^{-1} U \\
U & =\left[\begin{array}{ll}
0_{3 L} & I_{3 L}
\end{array}\right] .
\end{aligned}
$$

Finally, the magnetic-field estimate is given by removing the parts of the posterior mean and covariance relating to the magnetization. That is,

$$
\left[\begin{array}{c}
\hat{\mathcal{M}}_{k}\left(r_{k+1}^{(1)}\right) \\
\vdots \\
\hat{\mathcal{M}}_{k}\left(r_{k+1}^{(L)}\right)
\end{array}\right]=\left[\mu_{f * \mid y_{k}, y_{k}^{\eta}, y_{k+1}^{\eta}}\right]_{1: 3 L, 1: 3}
$$

and

$$
\Sigma_{\hat{\mathcal{M}}}=\left[\Sigma_{f^{*} f^{*} \mid y_{k}, y_{k}^{\eta}, y_{k+1}^{\eta}}\right]_{1: 3 L, 1: 3 L}
$$

Here the operator $[\mathbf{A}]_{i: j, m: l}$ cuts out the sub block corresponding to rows $i$ to $j$ and columns $m$ to $l$ of matrix $A$. The computational complexity of the learning process is approximately $(6 L)^{3}[18]$.

\footnotetext{
${ }^{1}$ Air is non-magnetic and thus has zero magnetization.
}

\section{EVALUATION}

To evaluate the performance of the proposed odometry estimation process when using the two magnetic-field models the following simulation experiments were conducted.

\section{A. Simulation setup and evaluation metrics}

Magnetic-field data was collected using a magnetometer within a volume of approximately $4 \mathrm{~m}^{3}$ in the room shown in Fig. 1. While collecting the data the position and orientation of the magnetometer was tracked by a high-end camera-based tracking system. A high-order dipole reference model was then fitted to the data. The field of the resulting reference model is shown in Fig. 4. Using the reference model, MonteCarlo simulations where an array moved according to three different trajectories through the field, were conducted. The three trajectories were:

1) An in-plane trajectory where the array moved without any rotations in a straight horizontal line, and with the $x y$-plane of the array aligned with the $x y$-plane of the reference model.

2) An out-of-plane trajectory where the array moved without any rotations in a straight vertical line perpendicular to the $x y$-plane of the array, and the $x y$-plane of the array was aligned with the $x y$-plane of the reference model.

3) A spiral trajectory where the array moved in an upward spiral with a constant radius and the $x$-axis of the array pointing in the tangent of the movement and the roll being zero.

The trajectories are shown in Fig. 4. In all the trajectories the displacement $\left\|\Delta p_{k}\right\|$ between the measurements was $100 \mathrm{~mm}$. For the spiral trajectory the change in orientation $\left\|\Delta q_{k}\right\|$ was $3^{\circ}$. Further, the geometry of the simulated array was the same as for the one shown in Fig. 2. That is, the array consisted of 30 magnetometers placed in a 6 times 5 grid with $64 \mathrm{~mm}$ and $55 \mathrm{~mm}$ spacing in the $x$ and $y$ axis directions, respectively. Moreover, the noise variance of the magnetometers was set to $\sigma_{e}^{2}=0.01^{2} \mu \mathrm{T}$. This corresponds to the noise level of the low-cost magnetometer RM3100 from PNI Sensor, US. ${ }^{2}$

Using the simulated array measurements the pose change of the array was estimated using (3) and the root-mean-squareerrors (RMSE)

$$
\operatorname{RMSE}\left\{\Delta \hat{p}_{k}\right\}=\sqrt{\frac{1}{M} \sum_{m=1}^{M}\left\|\Delta \hat{p}_{k}^{(m)}-\Delta p_{k}\right\|^{2}}
$$

and

$$
\operatorname{RMSE}\left\{\Delta \hat{q}_{k}\right\}=\sqrt{\frac{1}{M} \sum_{m=1}^{M}\left\|\Delta \hat{q}_{k}^{(m)}-\Delta q_{k}\right\|^{2}},
$$

were calculated. Here, the superscript $m$ denotes the simulation iteration at which the estimate was calculated and $M$ is

\footnotetext{
${ }^{2}$ Data sheet available at: https://www.pnicorp.com/wp-content/uploads/ RM3100-Breakout-Board-Product-Sheet.pdf
} 


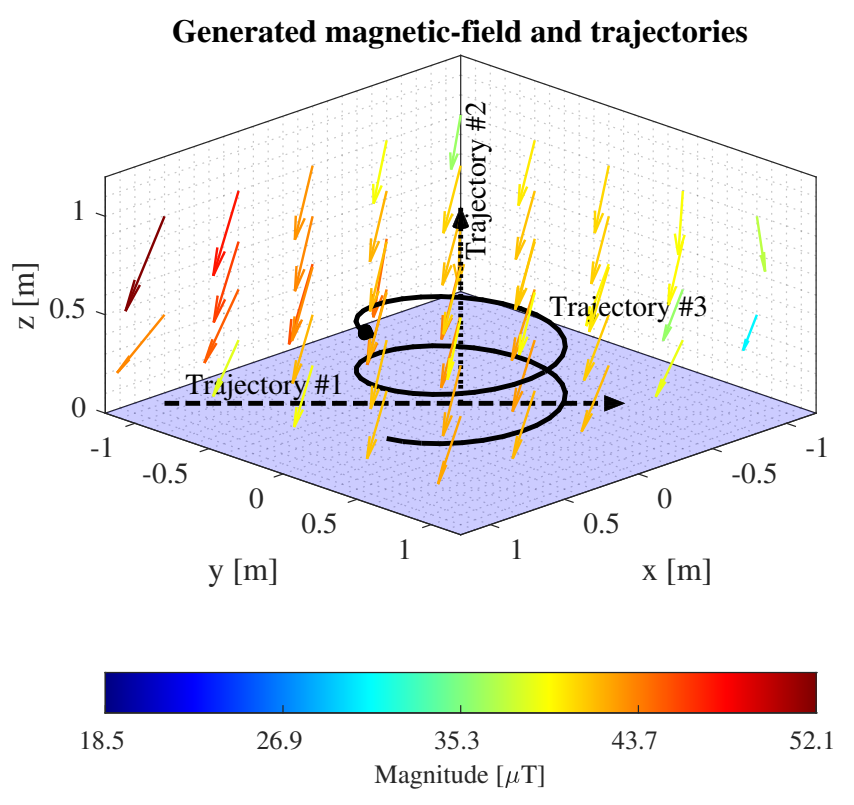

Fig. 4. Illustration of the magnetic-field and trajectories used in the simulations. The field model is based on real measurements to which a high-order dipole model has been fitted.

the total number of iterations in the Monte Carlo simulation. Furthermore, the mean values

$$
\bar{\Sigma}_{\Delta p_{k}}=\frac{1}{M} \sum_{m=1}^{M}\left[\Sigma_{\hat{x}_{k}}^{(m)}\right]_{1: 3,1: 3}
$$

and

$$
\bar{\Sigma}_{\Delta q_{k}}=\frac{1}{M} \sum_{m=1}^{M}\left[\Sigma_{\hat{x}_{k}}^{(m)}\right]_{4: 6,4: 6}
$$

of the uncertainties estimated by the estimator were computed. Moreover, as a measure of how much the field varied across the array the signal-to-noise ratio (SNR)

$$
\mathrm{SNR}_{k}=\frac{\frac{1}{3 L} \sum_{i=1}^{L}\left\|\mathcal{M}_{k}\left(d^{(i)}\right)-\overline{\mathcal{M}}_{k}\right\|^{2}}{\sigma_{e}^{2}}
$$

where

$$
\overline{\mathcal{M}}_{k}=\frac{1}{3 L} \sum_{i=1}^{L} \mathcal{M}_{k}\left(d^{(i)}\right)
$$

was calculated.

When learning the magnetic-field models the following settings were used. In the case of the polynomial model the only hyperparameter is the polynomial order $n$, which was automatically selected using AIC's method with a maximum allowed polynomial order $n=4$. In the case of the Gaussian process model all hyperparameters except the noise variance $\sigma_{e}^{2}$ were set to fixed values. The values $\sigma_{f}=5 \mu \mathrm{T}, \ell=0.15$ $\mathrm{m}$, and $\sigma_{\text {lin }}=15 \mu \mathrm{T}$ were used as they gave the overall best estimation performance. The noise variance $\sigma_{e}^{2}$ was estimated by maximizing the marginalized likelihood function as described in [6]. Noteworthy, theoretically it is possible to estimate all hyperparameters from the marginalized likelihood function, but in practice it prove to be difficult as the marginalized likelihood function contains multiple local optima.

\section{B. Results and discussion}

The results from the Monte Carlo simulations are shown in Fig 5-7. From the figures it can be seen that the estimation accuracy is highly dependent on the SNR. At a high SNR the displacement and orientation change can be estimated with an accuracy of $1-3 \mathrm{~mm}$ and $0.1-0.2^{\circ}$, respectively. This corresponds to a few percentage of the true pose change. Further, it can be seen that the polynomial and Gaussian process models perform approximately equally well, except for trajectory \#2 (the out-of-plane trajectory), where the Gaussian process model performs poorly for all SNR; the poor performance is likely due to the flexibility of the Gaussian process model, which makes it worse at extrapolating than the polynomial model. Moreover, when the Gaussian process model is used, the proposed odometry estimation process generally becomes overconfident in the accuracy of the estimated pose change. When the polynomial model is used there is a good agreement between the estimated and true uncertainty in the pose change.

Gaussian process models is the state-of-the-art technique for representing the magnetic-field map in magnetic-field SLAM solutions [5]. However, the presented results indicate that different magnetic-field models should be used for the odometry and mapping process if the proposed magneticfield odometry estimation process are to be combined with existing magnetic-field SLAM solutions. Considering that the polynomial model is a linear model of small dimension, the computational complexity of adding the odometry estimation process to existing magnetic-field SLAM solutions is low (approximately $n^{4} L^{2}$ floating point operations) and should not hinder real-time implementations.

\section{Conclusions \& Future Research}

A model-based magnetic-field odometry estimation process has been presented. Further, the performance of the proposed odometry estimation process when using a polynomial model and a Gaussian process model to describe the local magneticfield has been evaluated. The evaluation results shows that at high SNR the pose change can be estimated with an error of less than a few percentage of the true pose change. Moreover, the evaluation shows that with the polynomial model the uncertainty of the estimate can also be consistently predicted. Thus the proposed magnetic-field odometry technique constitutes a complimentary source of navigation information that can be used to reduce the position error growth rate of inertial navigation systems or other dead-reckoning systems. This is done by providing accurate and reliable odometry information when passing by magnetized objects, such as metal structures inside walls. When comparing the polynomial model and Gaussian process model, in general the polynomial model provides the highest odometry accuracy and the most consistent uncertainty estimates. Furthermore, the learning of the polynomial model has much lower computational complexity than the Gaussian 

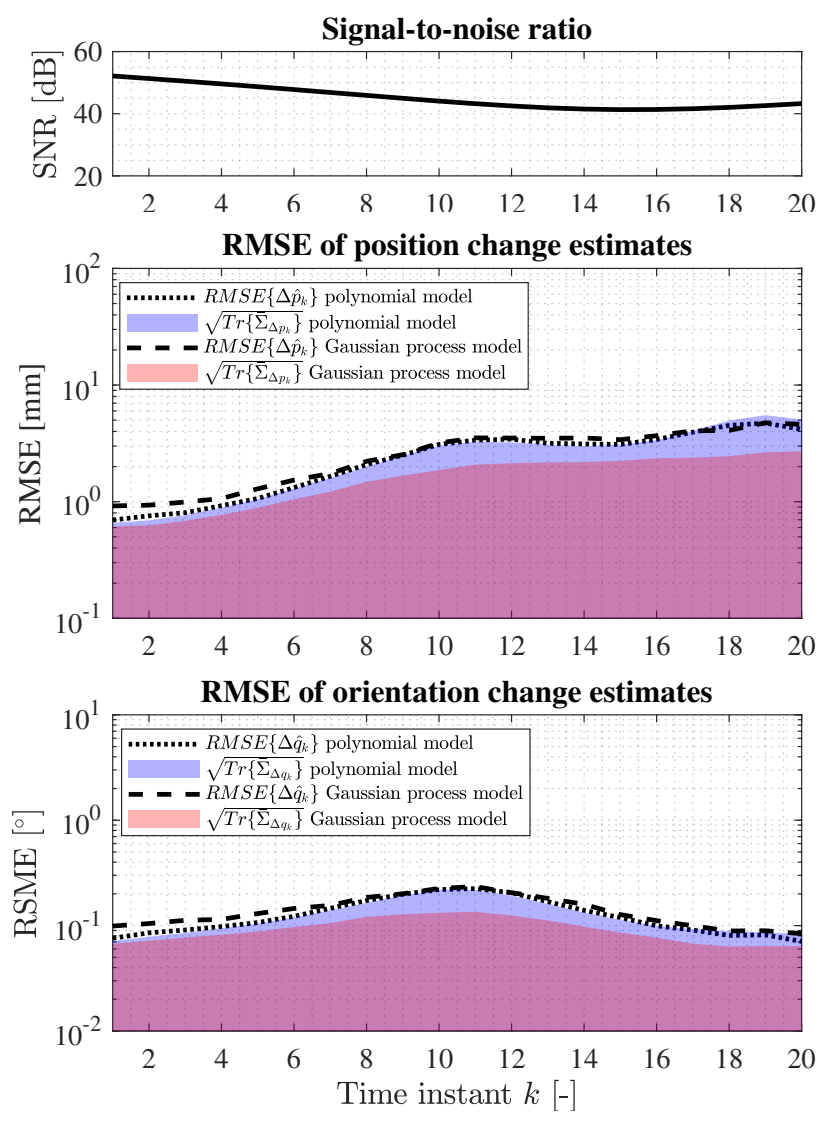

Fig. 5. Estimation error for trajectory \#1, i.e., the in-plane trajectory.

process model, which makes it more suitable for usage in real-time applications. Our future research will be focused on developing methods for tight integration between model-based magnetic-field odometry and inertial navigation systems.

\section{ACKNOWLEDGMENT}

This work has been funded by the Security Link project FOI-2010-1753:4 Magnetic-Field based Speed Aided Inertial Navigation and the Swedish Research Council project 202004253 Tensor-field based localization.

\section{REFERENCES}

[1] M. Angermann, M. Frassl, M. Doniec, B. J. Julian, and P. Robertson, "Characterization of the indoor magnetic field for applications in localization and mapping," in Int. Conf. on Indoor Positioning and Indoor Navigation (IPIN), Sydney, NSW, Australia, Nov. 2012.

[2] T. N. Lee and A. J. Canciani, "MagSLAM: Aerial simultaneous localization and mapping using earth's magnetic anomaly field," Navigation, Mar. 2020.

[3] H. F. Rice, V. Benischek, and L. Sczaniecki, "Application of atom interferometric technology for GPS independent navigation and time solutions," in IEEE/ION Position, Location and Navigation Symposium (PLANS), Monterey, CA, Jun. 2018.

[4] P. Robertson, M. Frassl, M. Angermann, M. Doniec, B. J. Julian, M. Garcia Puyol, M. Khider, M. Lichtenstern, and L. Bruno, "Simultaneous localization and mapping for pedestrians using distortions of the local magnetic field intensity in large indoor environments," in Int. Conf. on Indoor Positioning and Indoor Navigation (IPIN), Montbeliard, France, Oct. 2013.
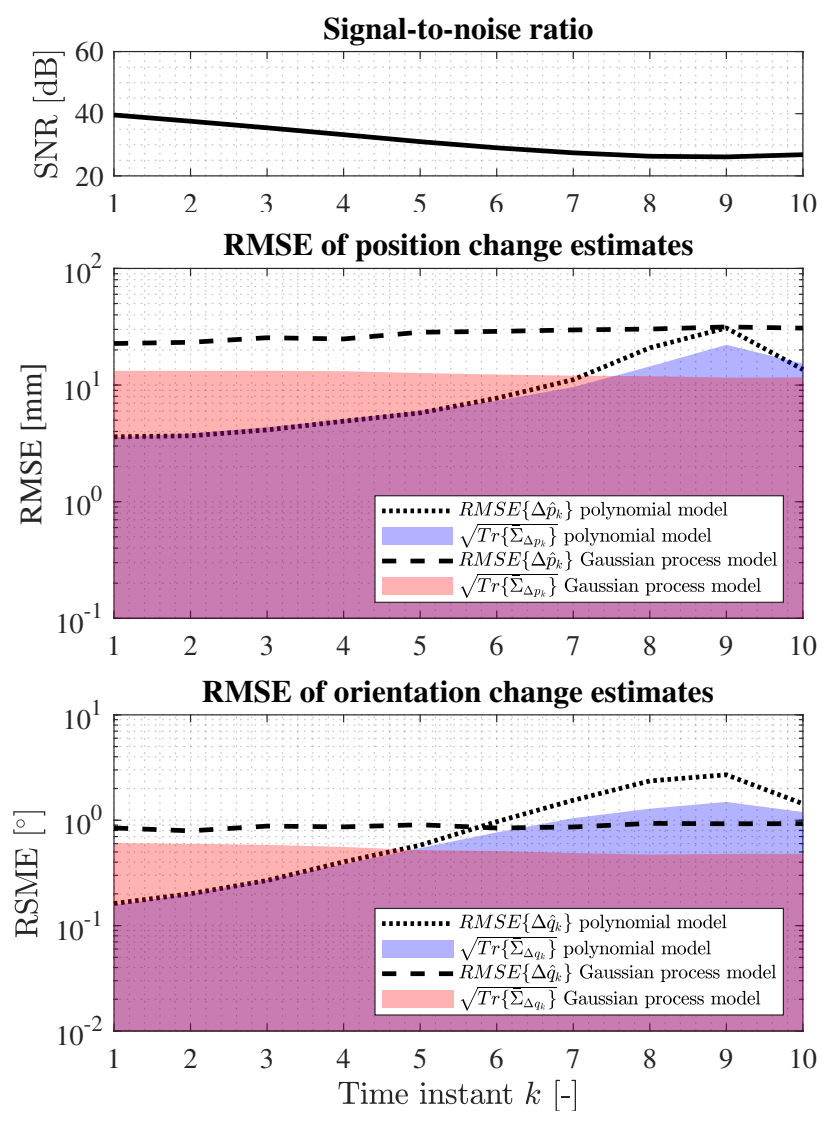

Fig. 6. Estimation error for trajectory \#2, i.e., the out-of-plane trajectory.

[5] M. Kok and A. Solin, "Scalable magnetic field SLAM in 3D using gaussian process maps," in International Conference on Information Fusion (FUSION), Cambridge, UK, Jul. 2018.

[6] N. Wahlström, M. Kok, T. B. Schön, and F. Gustafsson, "Modeling magnetic fields using Gaussian processes," in IEEE Int. Conf on Acoustics, Speech and Signal Processing (ICASSP), Vancouver, BC, May 2013.

[7] D. Vissiere, A. Martin, and N. Petit, "Using distributed magnetometers to increase IMU-based velocity estimation into perturbed area," in 46th IEEE Conf. on Decision and Control, New Orleans, LA, Dec. 2007.

[8] E. Dorveaux, T. Boudot, M. Hillion, and N. Petit, "Combining inertial measurements and distributed magnetometry for motion estimation," in Proc. of American Control Conf., San Francisco, CA, Jun. 2011.

[9] E. Dorveaux and N. Petit, "Presentation of a magneto-inertial positioning system: navigating through magnetic disturbances," in Int. Conf. on Indoor Positioning and Indoor Navigation (IPIN), Guimaraes, Portugal, Sep. 2011.

[10] C. I. Chesneau, M. Hillion, and C. Prieur, "Motion estimation of a rigid body with an EKF using magneto-inertial measurements," in Int. Conf. on Indoor Positioning and Indoor Navigation (IPIN), Alcala de Henares, Spain, Oct. 2016.

[11] M. Zmitri, H. Fourati, and C. Prieur, "Improving inertial velocity estimation through magnetic field gradient-based extended Kalman filter," in Int. Conf. on Indoor Positioning and Indoor Navigation (IPIN), Pisa, Italy, Oct. 2019.

[12] M. Zmitri, H. Fourati, and C. Prieur, "Magnetic field gradient-based ekf for velocity estimation in indoor navigation," Sensors, vol. 20, no. 20, 2020.

[13] — , "Bilstm network-based extended kalman filter for magnetic field gradient aided indoor navigation," IEEE Sensors Journal, vol. 10.1109/JSEN.2021.3091862, 2021.

[14] I. Skog, G. Hendeby, and F. Gustafsson, "Magnetic odometry - a modelbased approach using a sensor array," in Int. Conf. on Information Fusion (FUSION), Cambridge, UK, Jul. 2018. 

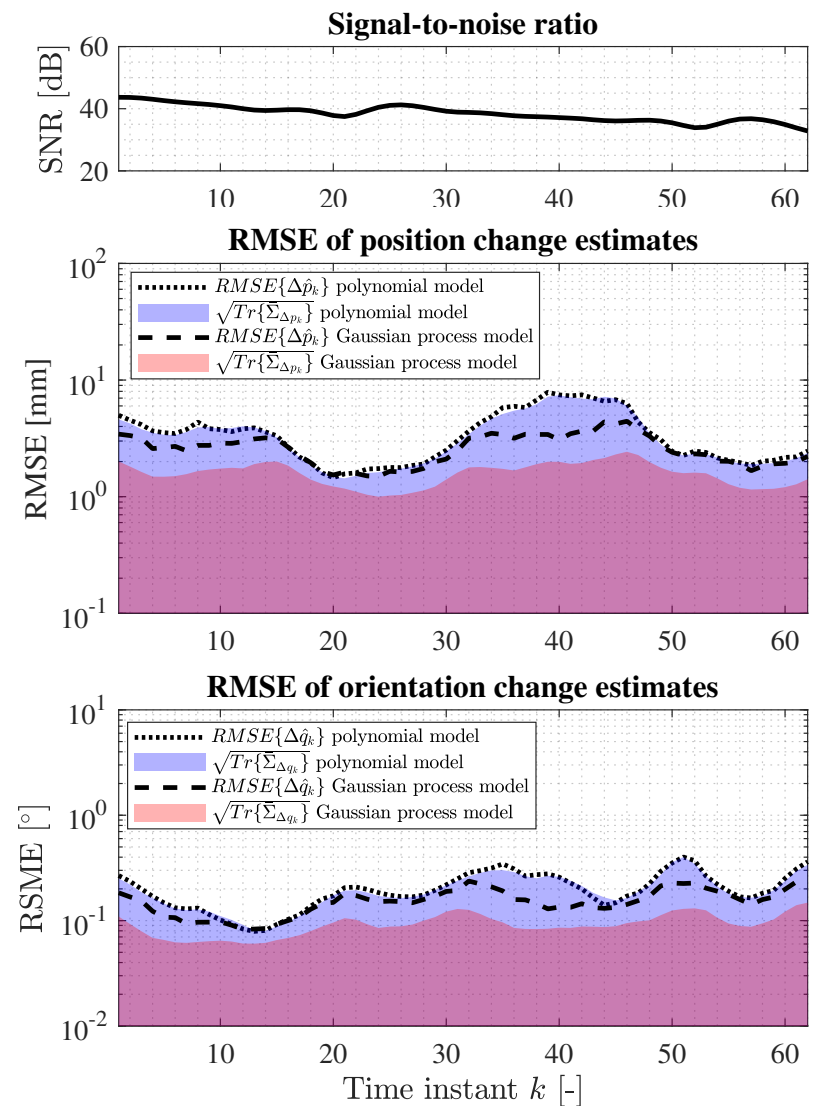

Fig. 7. Estimation error for trajectory \#3, i.e., the spiral trajectory.

[15] A. Marhaban and M. Saripan, "Review of visual odometry: types, approaches, challenges, and applications." SpringerPlus, vol. 5, no. 1897, Oct. 2016.

[16] S. M. Kay, Fundamentals of Statistical Signal Processing: Estimation Theory. Prentice Hall, 1993.

[17] L. Ljung, System Identification - Theory For the User, 2nd ed. Prentice-Hall, 1999.

[18] C. E. Rasmussen and C. K. I. Williams, Gaussian Processes for Machine Learning. MIT Press, 2006. 